\title{
Analysis of Crossover Operators for Cluster Geometry Optimization
}

\author{
Francisco B. Pereira \\ Instituto Superior de Engenharia de Coimbra \\ Portugal
}

\begin{abstract}
We study the effectiveness of different crossover operators in the global optimization of atomic clusters. Hybrid approaches combining a steady-state evolutionary algorithm and a local search procedure are state-of-the-art methods for this problem. In this paper we describe several crossover operators usually adopted for cluster geometry optimization tasks. Results show that operators that are sensitive to the phenotypical properties of the solutions help to enhance the performance of the optimization algorithm. They are able to identify and recombine useful building blocks and, therefore, increase the likelihood of performing a meaningful exploration of the search space.
\end{abstract}

\section{Introduction}

An atomic or molecular cluster is composed by a set of particles and it may present distinct physical properties from those of a single molecule or bulk matter. Estimating its most relevant properties has immediate relevance in many areas, ranging from protein structure prediction to the study of the influence of stratospheric clouds in ozone destruction. Also, a proper understanding of cluster properties is crucial for the field of nanotechnology.

The interactions among the particles that compose the cluster may be described by a multidimensional function, designated as Potential Energy Surface (PES), whose knowledge is mandatory in the theoretical study of the properties of a given chemical system (Stillinger, 1999). However, finding an organization for the particles that corresponds to the lowest potential energy turns out to be a NP- hard task (Wille \& Vennik, 1985). As this is a critical piece of information, stochastic global optimization algorithms have been increasingly used to discover the global minimum of the PES or, at the least, a set of low energy local minima. For systems with many particles, experiments are usually performed on model functions based on the sum of all pair potentials (i.e., functions that depend only on the distance between every pair of particles that compose the aggregate). The Morse 
function has been widely adopted as it accurately models both long-range interactions, such as those that appear in alkali metal clusters, and short range interactions arising in, e.g., $\mathrm{C}_{60}$ molecules (Morse, 1929).

Cluster geometry optimization is also relevant for global optimization, as it provides difficult instances for benchmarking new algorithms. Since the early 1990's Evolutionary Algorithms (EAs) have been increasingly applied to this task (Deaven \& Ho, 1995; Doye et al., 2004; Grosso et al., 2007; Hartke, 2001; Johnston, 2003; Pereira \& Marques, 2009; Zeiri, 1995). State-of-the-art methods usually combine a global evolutionary component with a gradient-driven local search procedure. A real valued representation and specific genetic operators help to enhance the effectiveness of the algorithm. In what concerns crossover, the Cut and Splice operator (C\&S) was proposed in 1995 by (Deaven \& Ho, 1995) and, since then, it has been adopted by most of the evolutionary approaches for cluster geometry optimization. C\&S is sensitive to the semantic properties of a solution and therefore is able to perform a meaningful recombination of the parents. Recently we proposed Generalized Cut and Splice crossover (GenC\&S), an extension that attempts to remove some limitations that are linked to the application of C\&S (Pereira et al., 2008). An empirical locality analysis was performed with both operators and confirmed that GenC\&S exhibits a behavior that is more in accordance to what is expected from crossover. GenC\&S was recently applied to argon clusters and it was able to discover all putative global optima up to 78 atoms (Marques et al., 2008).

In this paper we apply both $\mathrm{C} \& \mathrm{~S}$ and GenC\&S operators to several difficult instances of short-ranged Morse clusters to gain a deeper insight of their optimization abilities. A detailed analysis of the results will help to understand why GenC\&S is more effective for cluster geometry optimization.

The structure of the paper is the following: in section 2 we present the potential function used to model Morse clusters. Section 3 comprises the presentation of the optimization algorithm used in the experiments, including a detailed description of the crossover operators. The main results are presented and discussed in section 4 . Finally, section 5 gathers the main conclusions.

\section{Morse Potential}

The energy of Morse clusters is obtained by summing the Morse potentials describing all pair-wise interactions that occur in the aggregate (Morse, 1929):

$$
V_{\text {Morse }}=\varepsilon \times \sum_{i}^{N-1} \sum_{j>i}^{N}\left(e^{-2 \beta\left(r_{i j}-r_{0}\right)}-2 e^{-\beta\left(r_{i j}-r_{0}\right)}\right)
$$

In equation (1), $r_{i j}$ stands for the Euclidean distance between atoms $i$ and $j, \varepsilon$ is the bond dissociation energy, $r_{0}$ is the equilibrium bond length and $\beta$ is the range exponent of the 
potential. We adopt the scaled version of the Morse potential with non-atom specific interactions and, therefore, both $\varepsilon$ and $r_{0}$ are set to 1 (Doye \& Wales, 1997). The only adjustable parameter is then $\beta$, which determines the range of interparticle forces. We set $\beta=14$, corresponding to a short-range interaction. Discovering the global minimum is particularly hard this kind of instances, as the search landscape is extremely rough and it contains a huge number of local minima (Doye et al., 2004). Furthermore, the global minima of instances with a different number of atoms correspond to structures with distinct structural organization and properties, preventing the application of biased methods (Doye \& Wales, 1997; Grosso et al., 2007).

\subsection{Related Work}

An historical description of the application of EAs to cluster geometry optimization problems can be found in (Hartke, 2004). In this section we briefly highlight the most relevant contributions, focusing our attention on Morse clusters.

Doye and Wales were the first ones to apply an optimization algorithm to Morse clusters. They developed a basin-hopping method (it combines a Monte Carlo algorithm with local search) and were able to discover all but 12 of the putative global optima for short-ranged Morse clusters up to 80 atoms (Doye \& Wales, 1997). Johnston proposed the first hybrid EA for this problem. It was applied to short and medium range Morse clusters and discovered all putative global optima until 50 atoms (Johnston, 2003). In 2002, Locatelli and Schoen proposed population-basin hopping with a two-phase local optimization (Locatelli \& Schoen, 2002). This hybrid method proved to be extremely effective in discovering all putative global optima of Morse clusters until 80 atoms (Grosso et al., 2007). The shortcoming is this approach is that the first phase of local search requires the specification of a set of parameters that are problem dependent and therefore it cannot be easily extended to situations where the global optimum is unknown. Two recent papers describe approaches to remove the bias from the first phase of local search (Cassioli et al., 2208; Pereira \& Marques, 2008). In 2009, Pereira and Marques proposed a simple hybrid EA that is able to discover all putative global optima for short-ranged Morse clusters until 80 atoms (Pereira \& Marques, 2009). This is the first completely unbiased population-based method that was able to succeed in this task. Its effectiveness is related to the adoption of appropriate diversity measures that maintain the diversity in the population.

\section{Hybrid Optimization Algorithm}

The optimization framework adopted in this work combines a steady-state EA and a local search procedure. It is well-known from previous studies that hybrid approaches are particularly effective in discovering high quality solutions for cluster geometry 
optimization problems (Deaven \& Ho, 1995; Doye et al., 2004; Hartke, 2001; Johnston, 2003). On the one hand, the EA is able to efficiently sample large areas of the search space. On the other hand, a local search method that relies on first order derivative information, effectively guides search into the nearest local optimum. We rely on the Broyden-FletcherGoldfarb-Shanno method (L-BFGS), a quasi-Newton conjugate gradient procedure (Liu \& Nocedal, 1989). L-BFGS is applied to every new solution generated by the EA.

\subsection{Evolutionary Algorithm}

A straightforward steady-state model is used. Unlike generational EAs, in this model populations overlap and descendants compete for survival with existing solutions. In each iteration, a sequence of steps are performed: i) tournament selection chooses two parents that will be used to create the offspring; ii) crossover and mutation are applied to the selected individuals (see next section for details about the genetic operators); iii) Offspring are locally optimized and evaluated; iv) replacement rules are applied to determine if the offspring will be allowed to join the population.

There are two main driving forces that regulate the replacement of solutions: quality and diversity. A distance measure must be defined to estimate the similarity between to solutions. In this work we rely on the center of mass distance, a measure that is based on the distance of atoms to the cluster's center of mass (Grosso et al., 2007). Results reported in (Grosso et al., 2007; Pereira \& Marques, 2009) confirm that it is effective in accessing the similarity of possible solutions for Morse instances. In general terms, a descendant D is allowed to enter the population if one of the following conditions is satisfied (consult (Lee et al., 2003) for a detailed description of the replacement strategy):

i) D is similar to a solution $X$ that belongs to the current population and D is better than $\mathrm{X}$. If this happens, $\mathrm{D}$ replaces $\mathrm{X}$;

ii) $\mathrm{D}$ is different from all solutions currently in the population. In this case, it replaces the worst solution $\mathrm{X}$, providing that $\mathrm{D}$ is better than $\mathrm{X}$.

The application of this replacement strategy requires the definition of a parameter, $d_{\text {min }}$, specifying the minimum allowed distance between any two solutions that simultaneously belong to the population.

\subsubsection{Representation and Genetic Operators}

Each possible solution encodes the location of the particles that compose the cluster. For an instance with $\mathrm{N}$ atoms, it encodes $3 \times \mathrm{N}$ real values specifying the Cartesian coordinates of each particle. The domain for all variables is $\left[0, \mathrm{~N}^{1 / 3}\right]$ (Johnston, 2003). Only interatomic distances larger than 0.5 are allowed, since the potential becomes too repulsive is two atoms approach too much. Figure 1 exemplifies the structure of the chromosome. 


\section{\begin{tabular}{|l|l|l|l|l|l|l|l|l|l|}
\hline$x$ & $y$ & $z$ & $x$ & $y$ & $z$ & $\ldots$ & $x$ & $y$ & $z$ \\
\hline
\end{tabular}}

$\begin{array}{l:l:l:l:}\text { Atom } 1 & \text { Atom } 2 & \text { Atom } N & \end{array}$

Fig. 1. Structure of a chromosome

Both crossover and mutation are applied to generate descendants. The main goal of this paper is to study the effect of different crossover operators in the results achieved by the optimization algorithm. $\mathrm{C} \& \mathrm{~S}$ is an operator that is sensitive to the semantic properties of individuals involved in a crossover operation (Deaven \& Ho, 1995). It selects sub-clusters of the parents and combines them in such a way that it increases the likelihood of preserving relevant features of promising solutions. Since its proposal, $C \& S$ has been adopted by many researchers that applied EAs to cluster optimization problems (Hartke, 2001; Johnston, 2003). Results confirm that it contributes to enhance the efficacy of the optimization algorithm. In detail, C\&S performs the following operations to generate descendant D1 from parents P1 and P2 (another descendant D2 is created just by swapping the roles played by $\mathrm{P} 1$ and $\mathrm{P} 2$ ):

1. Apply random rotations to $\mathrm{P} 1$ and $\mathrm{P} 2$;

2. Define a random horizontal cutting plane (parallel to the $x y$ plane) for P1. This plane splits $\mathrm{P} 1$ in two complementary parts (X atoms below the plane and $\mathrm{N}-\mathrm{X}$ atoms above it);

3. Define a horizontal cutting plane (parallel to the $x y$ plane) for $\mathrm{P} 2$, in such a way that $\mathrm{X}$ atoms stay below the plane and $\mathrm{N}-\mathrm{X}$ are above it;

4. Generate D1 and D2 by combining complementary parts of each one of the parents.

Special precautions are taken when merging sections from different parents to ensure that the distance between two atoms in D1 is never below 0.5. C\&S ensures that the contribution of each parent is composed by a subset of atoms that are close together (i.e., a sub-cluster) and, therefore, they will tend to have a low potential energy. Hopefully, these building blocks increase the likelihood of discovering promising for the problems being solved.

Recently we proposed GenC\&S, an extension of the original C\&S operator (Pereira et al., 2008). The most important difference between these two variants is that GenC\&S relaxes some constraints that exist in the original version of the operator (e.g., the requirement of defining a horizontal plane to determine which atoms are inherited from each one of the parents). It just considers distances between atoms to determine the subset of atoms from each parent that will be used to create a descendant. Additionally, GenC\&S does not require the application of random rotations to the parents involved in a crossover operation. Considering the optimization of a cluster with $\mathrm{N}$ atoms, parents P1 and P2 lead to the creation of descendant D1 after the following steps are performed:

1. Select a random atom $\mathrm{CP}$ (the cut point) in $\mathrm{P} 1$.

2. Select a random number $S \in[1, \mathrm{~N}-1]$. 
3. Create a list $\mathrm{L}_{\mathrm{P} 1}$ consisting of the $\mathrm{N}$ atoms that belong to $\mathrm{P} 1$. Items in $\mathrm{L}_{\mathrm{P} 1}$ are ordered according to an increasing distance from the location of CP.

4. Copy the first $S$ atoms from $L_{P 1}$ to $D 1$.

5. Create a list $\mathrm{L}_{\mathrm{P} 2}$ consisting of the $\mathrm{N}$ atoms that belong to $\mathrm{P} 2$. Items in $\mathrm{L}_{\mathrm{P} 2}$ are ordered according to an increasing distance from the location of $\mathrm{CP}$.

6. Remove from $\mathrm{L}_{\mathrm{P} 2}$ atoms that are too close (i.e., at a distance smaller than 0.5 ) to particles already copied to D1.

7. Copy the first (N-S) atoms from $\mathrm{L}_{\mathrm{P} 2}$ to $\mathrm{D} 1$.

8. If less than $\mathrm{N}$ atoms were copied to $\mathrm{D} 1$ then the individual is completed with particles placed at random locations.

The example from figure 2 illustrates a crossover situation between two individuals with 5 atoms. Although it is presented in a two-dimensional plane, it clearly describes the crossover operation that occurs in three dimensions. In panel a) the two parents are displayed. In this example CP is atom number 5 from $\mathrm{P} 1$ and $\mathrm{S}=3$. The dotted circle in $\mathrm{P} 2$ marks the location of atom 5 from P1 (the cut point). This position is needed later to determine which atoms from P2 will be passed to the descendant. In panel b) we present the atoms inherited by $\mathrm{D} 1$ from P1. The 3 closest atoms to the cut point were selected. In panel c) we display the overlapping between the atoms that were already copied to D1 and those that belong to P2. We consider that overlapping atoms are too close and therefore cannot belong to the same cluster. In this example, atoms 8 and 9 from P2 are too close to atoms that already belong to D1 (so they will not be inherited by the descendant, even though they are near the cup point). In panel d) we show the atoms from P2 that can be inherited by the descendant. Finally, in panel e) we present the final descendant after receiving the 2 remaining atoms from P2. This parent passed atoms 6 and 7 to D1, since they are the closest non-overlapping atoms to the cut point.

For completeness, in the next section we also present results obtained with standard uniform crossover. With this operator, parents are scanned left to right and, in each position, the descendant inherits the atom from one of the parents with equal probability. The operator ensures that the child does not receive atoms that are too close to each other. Uniform crossover is blind to the spatial distribution of atoms. By comparing its results with those achieved by $C \& S$ and GenC\&S we will gain insight into the relevance of developing crossover operators that are sensitive to the structure of the solutions.

Sigma mutation is adopted to modify the location of a particle inside the cluster. The new position is obtained by slightly perturbing each coordinate with a random value drawn from a Gaussian distribution with mean 0 and standard deviation $\sigma$ ( $\sigma$ is a parameter of the algorithm). 


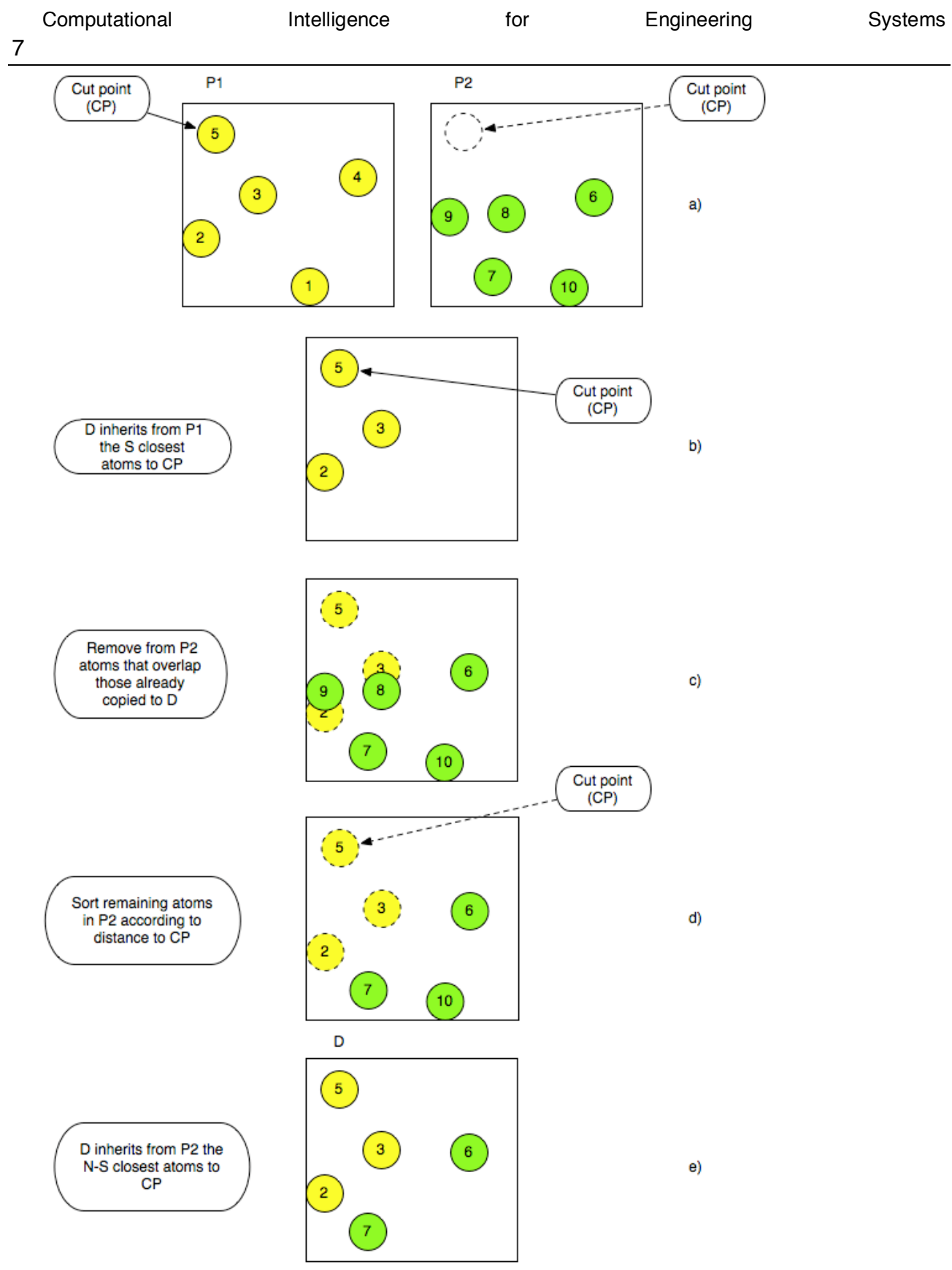

Fig. 2. Application of Generalized Cut and Splice crossover between two clusters. In this example $\mathrm{N}=5$ and Cut size=3. 


\section{Results and Discussion}

The performance of the hybrid EA with different crossover operators is accessed on a selected subset of short-ranged Morse instances. More specifically we selected the instances with the following number of atoms $\mathrm{N}=\{30,38,47,61,68\}$. This set includes examples that are considered to be difficult to optimize (see, e.g., (Grosso et al., 2007; Pereira \& Marques, 2009)).

The settings of the algorithm are the following: Number of runs: 30; Population size: 100; Evaluations: 5,000,000; Tournament size: 5; Crossover operators: \{Uniform, C\&S, GenC\&S $\}$; Crossover rate: $0.7 ; \sigma: 0.05 \times \mathrm{N}^{1 / 3}$; Mutation rate: $\{0,0.05,0.1\}$. Each iteration performed by the L-BFGS algorithm counts as one evaluation. Initial populations were always randomly generated.

Table 1. Optimization results obtained by C\&S crossover.

\begin{tabular}{|c|cc|cc|cc|}
\cline { 2 - 7 } \multicolumn{1}{c|}{} & \multicolumn{2}{c|}{ Mutation: 0.0} & \multicolumn{2}{c|}{ Mutation: 0.05} & \multicolumn{2}{c|}{ Mutation: 0.1} \\
\hline$N$ & Best & MBF & Best & MBF & Best & MBF \\
\hline 30 & $\mathbf{- 1 0 6 . 8 3 6}$ & -106.756 & $\mathbf{- 1 0 6 . 8 3 6}$ & -106.704 & $\mathbf{- 1 0 6 . 8 3 6}$ & -106.736 \\
\hline 38 & $\mathbf{- 1 4 4 . 3 2 1}$ & -142.568 & $\mathbf{- 1 4 4 . 3 2 1}$ & -142.439 & $\mathbf{- 1 4 4 . 3 2 1}$ & -142.617 \\
\hline 47 & -183.411 & -182.442 & -183.411 & -182.452 & -183.411 & -182.546 \\
\hline 61 & -249.586 & -246.093 & -248.612 & -246.209 & -248.612 & -247.157 \\
\hline 68 & -282.682 & -279.135 & -280.961 & -279.201 & -282.682 & -279.105 \\
\hline
\end{tabular}

Table 2. Optimization results obtained by GenC\&S crossover.

\begin{tabular}{|c|cc|cc|cc|}
\cline { 2 - 7 } \multicolumn{1}{c|}{} & \multicolumn{2}{c|}{ Mutation: 0.0} & \multicolumn{2}{c|}{ Mutation: 0.05} & \multicolumn{2}{c|}{ Mutation: 0.1} \\
\hline$N$ & Best & MBF & Best & MBF & Best & MBF \\
\hline 30 & $\mathbf{- 1 0 6 . 8 3 6}$ & -106.833 & $\mathbf{- 1 0 6 . 8 3 6}$ & -106.828 & $\mathbf{- 1 0 6 . 8 3 6}$ & -106.821 \\
\hline 38 & $\mathbf{- 1 4 4 . 3 2 1}$ & -143.999 & $\mathbf{- 1 4 4 . 3 2 1}$ & -143.366 & $\mathbf{- 1 4 4 . 3 2 1}$ & -143.428 \\
\hline 47 & $\mathbf{- 1 8 3 . 5 0 8}$ & -183.098 & -183.411 & -183.156 & $\mathbf{- 1 8 3 . 5 0 8}$ & -183.191 \\
\hline 61 & $\mathbf{- 2 4 9 . 5 8 8}$ & -248.745 & -249.586 & -248.741 & $\mathbf{- 2 4 9 . 5 8 8}$ & -248.833 \\
\hline 68 & $\mathbf{- 2 8 2 . 6 8 3}$ & -282.416 & $\mathbf{- 2 8 2 . 6 8 3}$ & -282.357 & $\mathbf{- 2 8 2 . 6 8 3}$ & -282.211 \\
\hline
\end{tabular}

Table 3. Optimization results obtained by Uniform crossover.

\begin{tabular}{|c|cc|cc|cc|}
\cline { 2 - 7 } \multicolumn{1}{c|}{} & \multicolumn{2}{c|}{ Mutation: 0.0} & \multicolumn{2}{c|}{ Mutation: 0.05} & \multicolumn{2}{c|}{ Mutation: 0.1} \\
\hline$N$ & Best & MBF & Best & MBF & Best & MBF \\
\hline 30 & $\mathbf{- 1 0 6 . 8 3 6}$ & -106.522 & $\mathbf{- 1 0 6 . 8 3 6}$ & -106.540 & $\mathbf{- 1 0 6 . 8 3 6}$ & -106.621 \\
\hline 38 & $\mathbf{- 1 4 4 . 3 2 1}$ & -141.633 & $\mathbf{- 1 4 4 . 3 2 1}$ & -141.512 & $\mathbf{- 1 4 4 . 3 2 1}$ & -141.414 \\
\hline 47 & -183.399 & -181.057 & -183.411 & -181.042 & -183.411 & -181.342 \\
\hline 61 & -249.153 & -245.402 & -247.593 & -245.773 & -249.153 & -245.920 \\
\hline 68 & -282.624 & -277.172 & -282.682 & -278.436 & -282.604 & -278.389 \\
\hline
\end{tabular}


In tables 1, 2 and 3 we present the results achieved by the three versions of the hybrid EA (one for each crossover operator). Columns Best show the potential energy of the best solution found, whilst columns $M F B$ present the mean best fitness (best solutions found averaged over 30 runs). In each table we present results achieved by the three different mutation rates. Entries in bold highlight experiments that were able to discover the putative optimum.

Results clearly show that both C\&S and GenC\&S outperform uniform crossover, confirming that operators sensitive to the phenotypic properties of a solution lead to an enhanced performance of the optimization algorithm. In what concerns the two specific operators for cluster geometry optimization, GenC\&S obtains better results showing that the removal of some artificial constraints helps the algorithm to effectively explore the search space. For the three largest instances, only GenC\&S allowed the hybrid EA to discover the global optimum.

Another interesting finding is that the hybrid EA is not sensitive to the variation of mutation rate. In fact, results even show that mutation is not needed at all, as the outcomes obtained without mutation are similar to those achieved when this operator is included in the EA. The explanation for this result is probably related to the application of local search that might be seen as some kind of directed mutation. When L-BFGS is applied it slightly modifies the location of the atoms (trying to find a more suitable configuration) and, therefore, its effect is similar to that of sigma mutation. Also, the EA includes a diversity mechanism, which naturally prevents the population from premature convergence.

In the charts from figure 3 we present the evolution of the MBF in tests performed with the Morse instance with 47 atoms (results for other instances follow the same trend): panel a) displays results obtained with $C \& S$ operator, panel b) concerns GenC\&S and panel c) shows the progress of MBF when uniform crossover is used. They support the previous analysis, as the evolution of the MBF is similar for the three mutation rates adopted. We performed a simple statistical analysis that confirms that there are never significant differences in the results achieved by the same crossover operator when different mutation rates are used (t-test with level of significance 0.01 ).

The chart from figure 4 displays the evolution of the MBF when different crossover operators are used. The Morse instance with 47 atoms and a mutation rate of 0.05 is considered, although the results are similar to those that occur with other instances and/or mutation rates. The performance ranking is clearly visible in this figure. The hybrid EA with uniform crossover exhibits that worst performance, whilst GEnC\&S is the operator that helps the optimization algorithm to achieve better results. $C \& S$ crossover is between the two extremes. Again, we performed a simple statistical analysis that, for the most cases, confirms the existence of significant differences in the results achieved by different crossover operators when applied to the same instance (t-test with level of significance $0.01)$. 

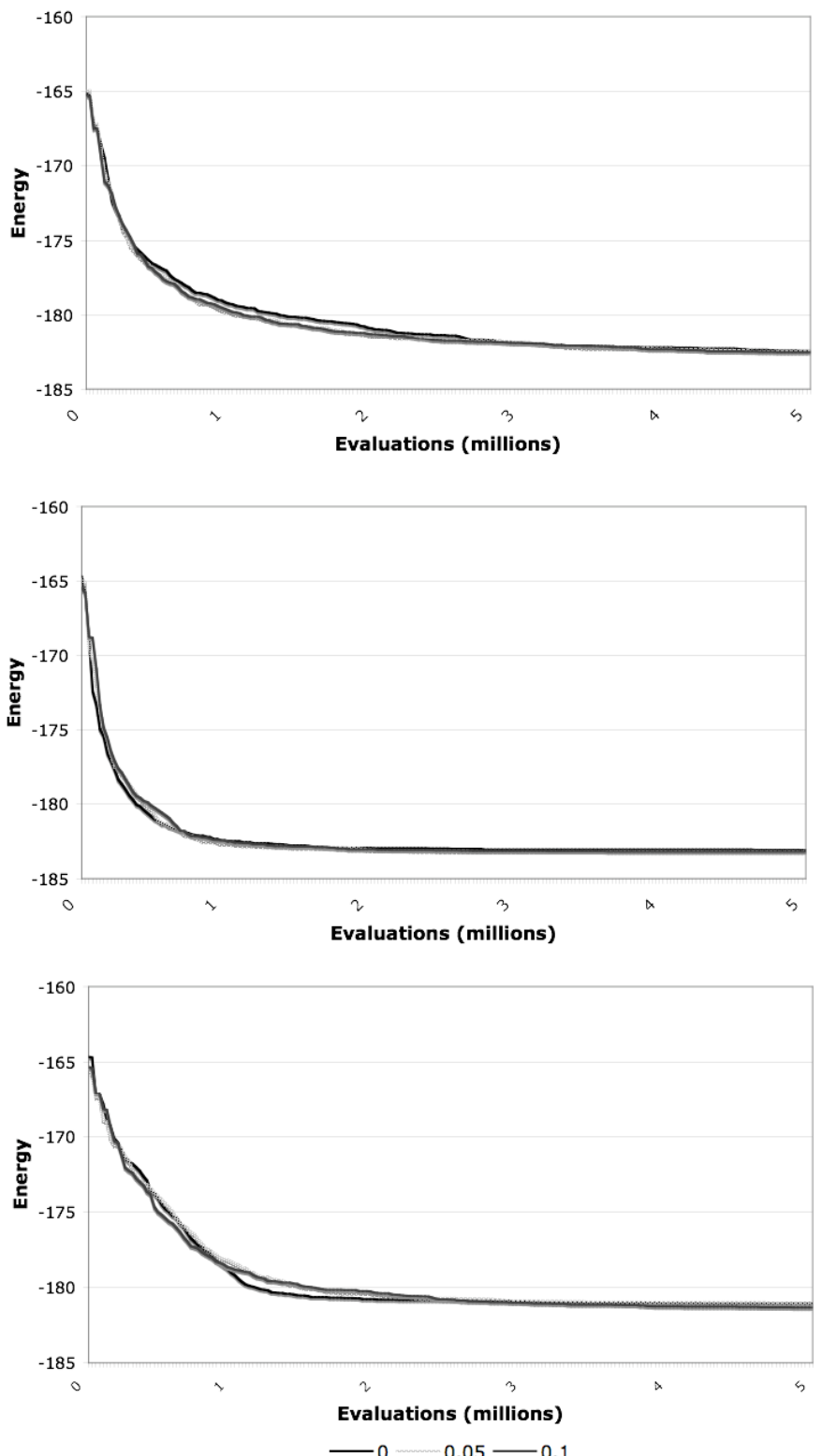

Fig. 3. Evolution of MBF in the optimization of the Morse cluster with 47 atoms. Three panels are presented: a) displays results obtained with $\mathrm{C} \& S$ crossover, b) with $G e n C \& S$ crossover and c) with Uniform crossover. 


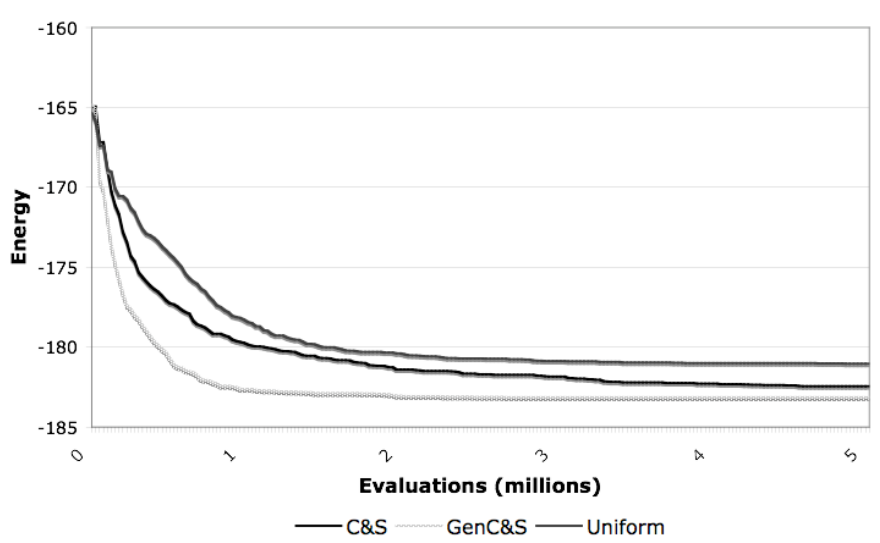

Fig. 4. Evolution of MBF in the optimization of the Morse cluster with 47 atoms in experiments performed with three different crossover operators. The mutation rate is 0.05 .

In table 4 we present a final set of results. Here we show (again for the instance with 47 atoms), for all combinations of crossover operators and mutation rates, the contribution of genetic operators to the discovery of new best solutions. Column Total displays how many new best solutions, (i.e., solutions that are better than any other discovered in that run) were found by the EA during the optimization. Results are totals for the 30 runs. Columns Mutation and Crossover show the proportion of new best solutions that were discovered thanks to, respectively, mutation and crossover (e.g., when C\&S crossover and a mutation rate of 0.05 are adopted, sigma mutation contributes to discover $91 \%$ of the new best solutions and C\&S helps to create $80 \%$ ).

Table 4. Contribution of the genetic operators for the discovery on new best solutions in the optimization of the Morse cluster with 47 atoms.

\begin{tabular}{|c|c|c|c|c|}
\hline & M_Rate & Total & Mutation & Crossover \\
\hline \multirow{3}{*}{ C\&S } & 0.0 & 538 & 0.00 & 1.00 \\
\cline { 2 - 5 } & 0.05 & 504 & 0.91 & 0.80 \\
\cline { 2 - 5 } & 0.1 & 494 & 1.00 & 0.72 \\
\hline \multirow{3}{*}{ GenC\&S } & 0.0 & 445 & 0.00 & 1.00 \\
\cline { 2 - 5 } & 0.05 & 436 & 0.92 & 0.96 \\
\cline { 2 - 5 } & 0.1 & 440 & 1.00 & 0.91 \\
\hline \multirow{3}{*}{ Uniform } & 0.0 & 445 & 0.00 & 1.00 \\
\cline { 2 - 5 } & 0.05 & 499 & 0.93 & 0.85 \\
\cline { 2 - 5 } & 0.1 & 517 & 1.00 & 0.83 \\
\hline
\end{tabular}

A brief perusal of the table reveals that the outcomes are similar for the three crossover operators. As expected, the increase in the mutation rate leads to a more frequent 
contribution of sigma mutation to the discovery of new best solutions. Crossover exhibits a high degree of participation in the creation of new best solutions. This is true for all operators (with a single exception, it is always above 80\%). Even though the proportions obtained for GenC\&S are slightly higher than those of both $\mathrm{C} \& \mathrm{~S}$ and uniform crossover, results suggest that the difference in performance is probably not related to the active participation of these operators in the exploration of the search space. The increased effectiveness of GenC\&S is a consequence of its ability to perform a meaningful recombination of the building blocks that compose the promising solutions chosen by selection.

\section{Conclusions}

Hybrid algorithms combining evolutionary methods and local search procedures are state-of-the-art techniques for cluster geometry optimization. A key component of the EA is the crossover operator that identifies and combines useful building blocks that may help to discover high quality solutions. In this paper we introduced three crossover operators that can be used in this problem and described a set of experimental results that helped to gain insight into the weaknesses and strengths of each one of the operators.

Results clearly show that operators that are sensitive to the semantic properties of the individuals they manipulate are better suited for this optimization task. Of all operators tested, GenC\&S crossover proved to be the most effective. This operator, not only is able to see the individual as a 3D cluster, but also removes some artificial constraints that existed in previous operators.

\section{Acknowledgments}

This work was supported by Fundação para a Ciência e Tecnologia, Portugal, under grant PTDC/QUI/69422/2006.

\section{References}

Cassioli, A., Locatelli, M. \& Schoen F. Global optimization of binary Lennard-Jones clusters. Optimization Methods and Software, online December 2008 (DOI: 10.1080/10556780802614101), 2008.

Deaven, D. \& Ho., K. Molecular geometry optimization with a genetic algorithm. Phys. Rev. Lett., (75):288-291, 1995 
Doye, J. P. K. \& Wales, D. Structural consequences of the range of the inter-atomic potential. a menagerie of clusters. J. Chem. Soc. Faraday Trans., (93):4233-4243, 1997.

Doye, J. P. K., Leary, M., Locatelli, M. \& Schoen, F. Global optimization of Morse clusters by potential energy transformations. Informs Journal on Computing, (16):371-379, 2004.

Grosso, A., Locatelli, M. \& Schoen, F. A population-based approach for hard global optimization problems based on dissimilarity measures. Math. Program. Ser. A, (110):373-404, 2007.

Hartke, B. Global geometry optimization of atomic and molecular clusters by genetic algorithms. In Proceedings of the Genetic and Evolutionary Computation Conference (GECCO-2001), pages 1284-1291. Morgan-Kaufmann, 2001.

Hartke, B. Application of evolutionary algorithms to global cluster geometry optimization. In Applications of Evolutionary Computation in Chemistry, Structure and Bonding, pages 33-53. Springer-Verlag, 2004.

Johnston, R. Evolving better nanoparticles: Genetic algorithms for optimising cluster geometries. Dalton Transactions, (22):4193-4207, 2003.

Lee, J., Lee, I.-H. \& Lee J. Unbiased global optimization of Lennard-Jones clusters for $\mathrm{n} \leq 201$ by conformational space annealing method. Phys. Rev. Lett., 91(8):080201.1$080201.4,2003$.

Locatelli, M. \& Schoen, F. Fast global optimization of difficult Lennard-Jones clusters. Comput. Optim. Appl., 21, 55-70, 2002.

Liu, D. C. \& Nocedal. J. On the limited memory method for large scale optimization. Mathematical Programming B, (45):503-528, 1989.

Marques, J., Pereira, F. B. \& Leitão, T. On the use of different potential energy functions in rare-gas cluster optimization by genetic algorithms : application to argon clusters. Journal of Physical Chemistry A, 112 (27), 6079-6089, 2008.

Morse, P. Diatomic molecules according to the wave mechanics. ii. vibrational levels. Phys. Rev., (34):57-64, 1929.

Pereira, F. B. \& Marques, J. A self-adaptive evolutionary algorithm for cluster geometry optimization. In Proceedings of the Eight International Conference on Hybrid Intelligent Systems, pages 678-683. IEEE Press, 2008.

Pereira, F. B., Marques, J., Leitao, T. \& Tavares, J. Efficient evolutionary algorithms for cluster optimization: A study on locality. In Advances in Meta-heuristics for Hard Optimization, pages 223-250. Springer-Verlag, 2008.

Pereira, F. B. \& Marques, J. A study on diversity for cluster geometry optimization. Evolutionary Intelligence (to appear), 2009.

Stillinger, F. Exponential multiplicity of inherent structures. Phys. Rev. E, 59, 48-51, 1999.

Wille, L. T. \& Vennik, J. Computational complexity of the ground-state determination of atomic clusters. J. Phys. A : Math. Gen. 18, 419-422, 1985.

Zeiri, Y. Prediction of the lowest energy structure of clusters using a genetic algorithm. Phys. Rev., (51):2769-2772, 1995. 